\title{
Daily Cannabis Users with Sickle Cell Disease Show Fewer Admissions than Others with Similar Pain Complaints
}

\author{
Susanna A. Curtis, ${ }^{1, *}$ Amanda M. Brandow, ${ }^{2}$ Michelle DeVeaux, ${ }^{3}$ Daniel Zeltermam, ${ }^{4}$ Lesley Devine, ${ }^{1}$ and John D. Roberts ${ }^{1}$
}

\begin{abstract}
Introduction: Previous studies have shown that cannabis use is common in adults with sickle cell disease (SCD), and that many patients report using cannabis to treat pain.

Methods: We performed a cross-sectional study of adults with SCD and compared daily users of cannabis with others using validated patient-reported measures of pain and quality of life as well as opioid and health care utilization.

Results: Daily cannabis users with SCD had worse pain episode severity scores than others ( $56.7 \mathrm{vs.} 48.8, p=0.02)$ yet had 1.8 fewer annual admissions ( $p=0.01$ ) and 1.2 fewer annual emergency room (ER) visits $(p=0.01)$, and similar amounts of opioids dispensed to others after matching for age, gender, SCD genotype, hydroxyurea use, and pain impact scores.

Conclusions: We show that people with SCD with more severe pain crisis are more likely to use daily cannabis, yet have lower rates of hospital admission and ER use as compared with others with similar disease severity and pain impact. Randomized controlled trials should be performed.
\end{abstract}

Keywords: sickle cell disease; pain; cannabinoid; cannabis

\section{Introduction}

Sickle cell disease (SCD) affects $>100,000$ adults in the United States, accounting for $>113,000$ annual hospital admissions, the majority for uncontrolled pain. ${ }^{1}$ Pain in SCD is complex. People with SCD can have acute pain and chronic pain, can have nocioceptive pain and neuropathic pain, and can develop peripheral and central sensitization to pain. Pain in patients with SCD is primarily treated with opioid medications, which are often not sufficient, and can create risk for abuse and overdose. Thus, there is a critical need for nonopioid treatments for pain in SCD.,

There is increasing evidence that cannabis or cannabinoids, the active chemical compounds in cannabis, may be an effective nonopioid treatment for pain. There is moderate quality evidence that cannabinoids are associated with a $30 \%$ reduction in neuropathic pain. ${ }^{4}$ There is also moderate quality evidence to support the use of cannabinoids in chronic pain. ${ }^{5}$ In 2017, the Committee on the Health Effects of Marijuana of the National Academies of Science, Engineering, and Medicine reported that there is "conclusive or substantial evidence that cannabis or cannabinoids are effective for the treatment of chronic pain in adults." Furthermore, states that enact medical cannabis laws show fewer opioid-related hospitalizations, reductions in opioid prescriptions for patients who receive both Medicaid and Medicare part D, and reductions in opioid overdose deaths. ${ }^{7-10}$

Cannabis is also becoming more accessible in the United States. As of the 2018 November elections there are now 34 states with medical marijuana laws, an additional 12 states with low-dose tetrahydrocannabinol cannabidiol laws, and 10 states with recreational

\footnotetext{
${ }^{1}$ Department of Internal Medicine, Yale Cancer Center, Yale School of Medicine, New Haven, Connecticut.

${ }^{2}$ Department of Pediatrics, Medical College of Wisconsin, Milwaukee, Wisconsin.

${ }^{3}$ Department of Early Clinical Development, Regeneron Pharmaceuticals, Tarrytown, New York.

${ }^{4}$ Department of Biostatistics, Yale School of Public Health, New Haven, Connecticut.

Presented in abstract form at The 59th Annual Meeting of the American Society of Hematology, Atlanta, GA, December 11, 2017.

*Address correspondence to: Susanna A. Curtis, Yale Cancer Center, Yale School of Medicine, 37 College Street, Office \#16, New Haven, CT 06510, E-mail: susanna.curtis@yale.edu
} 
cannabis laws. ${ }^{11}$ New evidence and new access are leading both physicians and patients to ask if medical marijuana could be used as a nonopioid treatment for pain in SCD, especially given its possible treatment of neuropathic pain.

Patterns of cannabis use in adults with SCD have been examined. In 2005, Howard et al. found that $36 \%$ of adults with SCD had used cannabis in the past 12 months, $52 \%$ for pain relief and $39 \%$ for improved mood. ${ }^{12}$ In $2000,4.6 \%$ of women and $38.3 \%$ of men in Jamaica reported using cannabis, and in $2004,19.4 \%$ of women and $64.6 \%$ of men reported using cannabis. ${ }^{13}$ In 2017, a 15-year retrospective review of urine toxicology studies of 72 patients found that $51 \%$ of patients tested positive for cannabis at some point during that time period. ${ }^{14}$ In $2018,42 \%$ of adults with SCD in the United States reported cannabis use in the past 2 years, the majority endorsed pain relief, and $79 \%$ of patients felt it allowed them to use less opioid medications. ${ }^{15}$ These studies show that cannabis use is common in adults with SCD, and that patients endorse pain relief from cannabis use. $^{12,15}$ It is notable that surveys of people with chronic pain from other causes also report high rates of cannabis use. ${ }^{16-18}$

That cannabis use is common in SCD and other diseases of pain suggests that it may have some effect in the treatment of pain. That people with SCD state that they use cannabis for pain relief as seen in our and other studies also suggests this. ${ }^{12,15}$ However, previous studies of cannabis use in SCD have not shown clear improvement in pain outcomes with cannabis use. Howard et al. compared cannabis users with nonusers and found no difference in numbers of pain episodes reported in the prior year. ${ }^{12}$ Knight-Madden et al. reported that cannabis use was not associated with "pain" in a logistic regression model after adjusting for age, gender, and year of the study. ${ }^{19}$ Ballas showed that patients with a history of a urine test positive for cannabis had a higher number of total hospital admissions during the 15-year time period than those who never had a positive test $(2443$ vs. $1602 p<0.05)$, but did not have higher levels of opioid use. ${ }^{14}$

Because all published studies of cannabis in people with SCD have been cross-sectional, it is not possible to establish a temporal relationship between cannabis use and pain outcomes. If all people with SCD are equally likely to use cannabis, the previous studies findings would suggest that cannabis use has no or a negative effect on pain outcomes or narcotic use. However, if people with more baseline pain are more likely to use cannabis, and cannabis is effective for pain, then those patients with more baseline pain who use cannabis may have pain outcomes or narcotic use similar to those with less baseline pain. A similar issue is seen in some cross-sectional studies of adults with SCD that show hydroxyurea use correlated with poor outcomes. ${ }^{20}$ Disease severity is associated with likelihood of using hydroxyurea and with likelihood of poor outcomes so disease severity is a confounder for the effect of hydroxyurea on other outcomes. We wondered if pain severity was confounding the potential analgesic effect of cannabis in SCD. We sought to examine the effects of cannabis use in SCD on acute and baseline pain outcomes and other quality-of-life outcomes and to adjust for possible confounding factors such as disease severity and hydroxyurea use.

\section{Methods}

\section{Subjects}

Subjects enrolled were adults (age $\geq 18$ years) with a diagnosis of SCD (HbSS, HbSC, $\mathrm{HbS} \beta+$, or $\mathrm{HbS} \beta 0$ ) who presented for regular scheduled clinic visits during the enrollment period. Patients were excluded if they had any complaint of acute pain or illness, were pregnant, or were unable to offer informed consent. Each subject was surveyed only once.

This study was conducted as approved by the Yale New Haven Hospital Institutional Review Board and informed consent was obtained before study procedures. Subjects were financially compensated for their participation.

\section{Patient-reported outcomes}

All patient-reported outcomes (PROs) were selfreported by patients using a pencil and paper instrument (or survey instrument). A survey designed by the investigators based on previous studies that asked whether patients had used cannabis in the past 30 days and, if so, how frequently (Supplementary Fig. S1). They were also given a list of options for why they chose to use cannabis, including for pain, anxiety, mood, sleep, appetite, to use less medications, to get high, and could select as many answers as they wanted.

Pain was assessed through the Adult Sickle Cell Quality of Life Measurement Information System (ASCQ-Me) domains for crisis pain frequency, crisis pain severity, and pain impact and Patient-Reported Outcomes Measurement Information System (PROMIS) domains pain quality nocioceptive pain and pain quality neuropathic pain 
and all items from each domain were completed. ${ }^{21-23}$ All domains have a reference population with a median score of 50 and a standard deviation (SD) of 10, the reference population for the ASCQ-Me domains was adults with SCD. The crisis pain frequency and crisis pain severity domains ask about crisis for the past 12 months and higher scores represent more severe disease. The pain impact domain asks about pain in the past 7 days and lower scores represent more severe pain impact. The higher scores on the PROMIS instruments for nocioceptive and neuropathic pain indicate a greater likelihood of pain being neuropathic or nocioceptive. ${ }^{21-24}$

Domains for other quality-of-life outcomes included instruments for ASCQ-Me domains for stiffness, sleep impact, emotional impact, and social impact and PROMIS domains Anxiety Form 8a, and Gastro Intestinal Distress Nausea and Vomiting (GI). All other ASCQ-Me domains ask about symptoms for the past 7 days except social functioning that asks about the past 30 days, and lower scores represent more severe disease. ${ }^{22}$ Higher scores indicate greater levels of anxiety or GI distress on the PROMIS instruments. ${ }^{25,26}$

\section{Clinical data}

Clinical information for each subject was obtained from the Yale New Haven Hospital electronic medical record. Hydroxyurea use was defined as patients with an active (recently filled) prescription for hydroxyurea at the time of enrollment. Transfusion use was defined as patients receiving either regular scheduled simple red blood cell transfusions or exchange transfusions at the time of enrollment. Total number of emergency room (ER) visits, admissions, and average length of stay in days at Yale New Haven Hospital from January 1, 2016 to December 31, 2016 for each individual patient were recorded. Total milligrams of opioids dispensed from January 1, 2016 to December 31, 2016 was obtained from the Connecticut Prescription Monitoring and Reporting System (PMPAware) database and converted to daily oral morphine equivalents (OME). ${ }^{27}$

\section{Statistical considerations}

Descriptive statistics were utilized to examine the demographics of patients. Reasons for use were compared between groups using a chi-squared test. Comparisons of PROs of three groups were done using a one-way analysis of variance (ANOVA) followed by pairwise comparisons. For comparisons of patient demographics, health care utilization, PROs, means, and standard deviations were calculated and were compared using Student's $t$-test for parametric data and medians and interquartile ranges were calculated and compared using the Wilcoxon rank-sum test for nonparametric data. Hospital admissions, length of stay in days, and ER visits of daily users of cannabis were compared with others using propensity matching for age, gender, genotype, hydroxyurea use, and 7-day pain impact score and a second time using age, gender, genotype, hydroxyurea use, and daily opioids dispensed in OME.

\section{Results}

We did not power our study to address

a primary hypothesis

We started this study with hypothesizes based on studies of the patterns and effects of cannabis and cannabinoid use in other diseases of pain. However, as no previous studies have had access to validated PROs specific for adults with SCD, there was no way to estimate potential findings or potential effect size. Thus, we enrolled a convenience sample of patients and did not power our study for any one hypothesis.

Patient's reported rates of cannabis use and reasons for using were similar to those seen in previous studies, and reasons did not vary between daily users and others

Fifty patients were enrolled from May 2017 to October 2017. Approximately $90 \%$ of patients offered enrollment agreed to participate; most refusals were due to subject time constraints. One patient's data were not used when she reported being in pain crisis later that day. Of the remaining 49 patients, 22 (45\%) reported cannabis use in the past 30 days (Fig. 1). Of those who had used cannabis in the past 30 days, $10(46 \%)$ reported daily use, 8 (36\%) reported weekly use, 3 (14\%) reported monthly use, and 1 (5\%) reported less than monthly use. Previous reports have shown that daily users more likely to use for medical reasons and infrequent users are more likely to use recreationally. ${ }^{28,29}$ Based on this information we compared self-reported reasons for using cannabis between daily users and all other users and found no significant differences (Fig. 2). Both groups selected pain relief as the most common reason for cannabis use.

Daily cannabis users had similar demographics to others and had similar rates of health care utilization but had worse pain episode severity than others We then examined pain outcomes (pain episode frequency, pain episode severity, pain impact, nocioceptive 


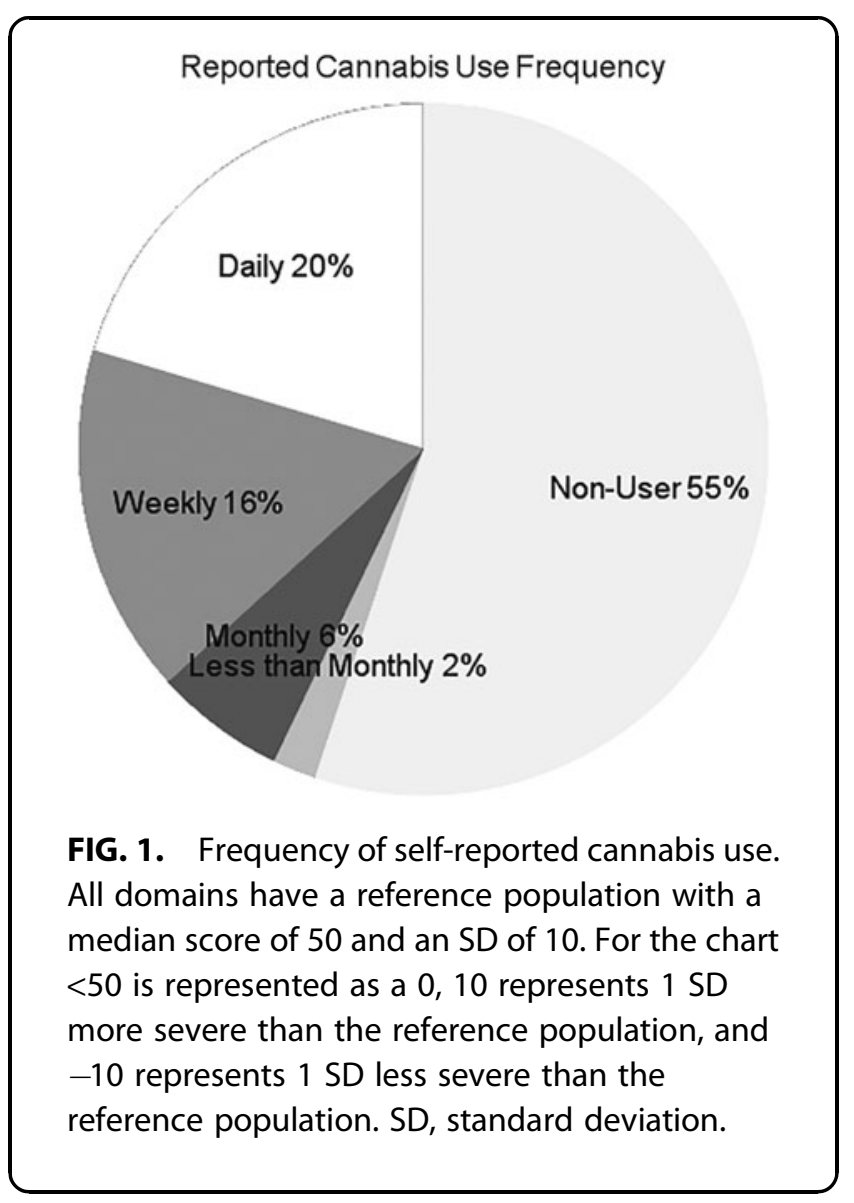

pain quality, and neuropathic pain quality), comparing daily users with infrequent (weekly, monthly, and less than monthly) users and with nonusers. There were no differences in pain episode frequency, pain impact, or nocioceptive and neuropathic pain quality seen between groups (Supplementary Fig. S2). Pain episode severity showed a trend $(p=0.058)$ of daily users having more severe pain than either infrequent cannabis users or nonusers who both had similar scores ( 56.7 vs. 48.4 vs. 48.9). As daily users appeared to be a distinct group based on pain scores and infrequent and non-cannabis users appeared similar we combined infrequent cannabis users and non-cannabis users into one group (others) for subsequent comparisons.

There were no differences in age, gender, hydroxyurea use, percentage receiving regular simple or exchange transfusions, or genotype frequency between daily users and others (Table 1). When daily users were compared with others they had higher pain episode severity scores (56.7 vs. $48.8, p=0.02$ ); other pain domains were similar (Fig. 3). They also had similar quality-of-life domain scores compared with others (Fig. 4). Daily users had similar rates of annual admissions, annual ER visits, and length of stay in days, as well as similar amounts of daily opioids dispensed in OME (Table 1).

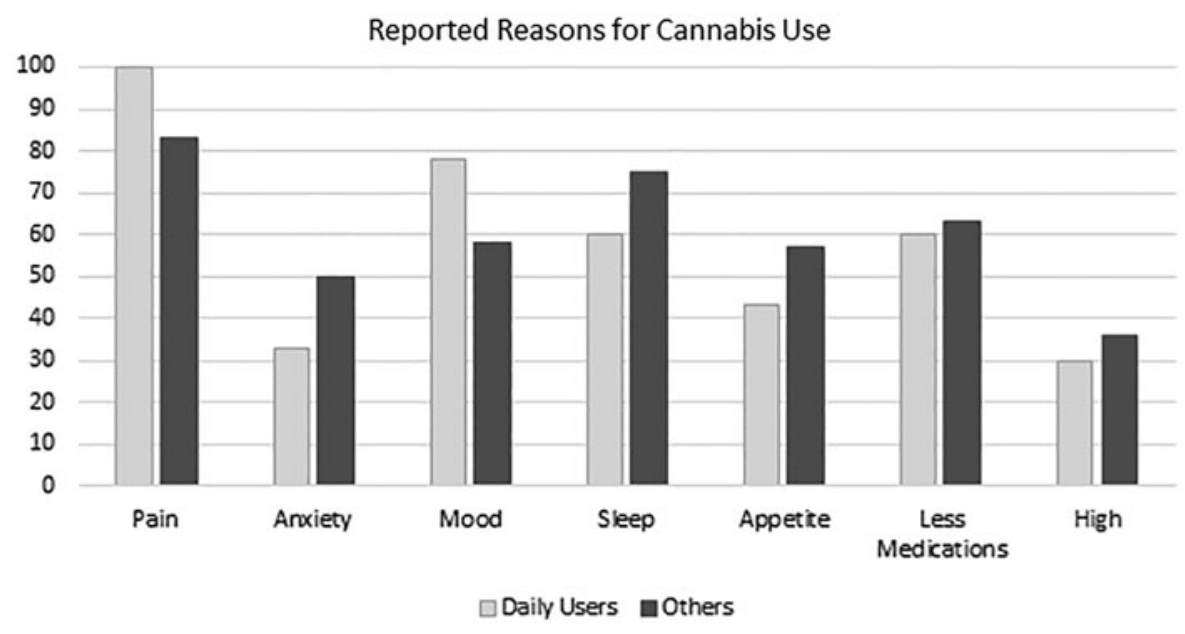

FIG. 2. Self-reported reasons for use among patients who endorsed cannabis use. Patients were allowed to endorse multiple reasons. All domains have a reference population with a median score of 50 and an SD of 10. For the chart $\angle 50$ is represented as a 0,10 represents 1 SD more severe than the reference population, and -10 represents 1 SD less severe than the reference population. 
Table 1. Clinical Outcomes of Daily Users Compared with Others

\begin{tabular}{|c|c|c|c|c|c|c|c|}
\hline & Daily users & Others & $p^{*}$ & $\begin{array}{c}\text { Coefficient propensity } \\
\text { match } 1\end{array}$ & $p$ & $\begin{array}{c}\text { Coefficient propensity } \\
\text { match } 2\end{array}$ & $p$ \\
\hline N & 10 & 39 & - & - & - & - & - \\
\hline Age, years (mean $\pm S D)$ & $34.3 \pm 14.7$ & $31.8 \pm 8.2$ & 0.6 & - & - & - & - \\
\hline Female, $\%$ & $50 \%$ & $60 \%$ & 0.7 & - & - & - & - \\
\hline Genotype, \% & $\begin{array}{l}\text { SS/S } \beta 0(70 \%) \\
\text { SC/S } \beta+(30 \%)\end{array}$ & $\begin{array}{l}\mathrm{SS} / \mathrm{S} \beta 0(68 \%) \\
\mathrm{SC} / \mathrm{S} \beta+(32 \%)\end{array}$ & 0.9 & - & - & - & - \\
\hline Hydroxyurea, \% & 30 & 33 & 0.7 & - & - & - & - \\
\hline Transfusion, \% & 30 & 33 & 0.8 & & & & \\
\hline Daily opioid use, OME median (quartiles) & $21.9(1.8 / 492.6)$ & $5.6(0.5 / 119.0)$ & 0.5 & $87.1(95 \% \mathrm{Cl}-292.6$ to 466.9$)$ & 0.7 & - $\quad-1$ & - \\
\hline Admissions in 2016 (mean \pm SD) & $1.1 \pm 1.5$ & $3.1 \pm 4.1$ & 0.2 & $-1.8(95 \% \mathrm{Cl}-3.2$ to -0.4$)$ & 0.01 & $-2.5(95 \% \mathrm{Cl}-4.5$ to -0.4$)$ & 0.02 \\
\hline Length of admission, days (mean \pm SD) & $4.2 \pm 1.6$ & $4.0 \pm 1.9$ & 0.7 & $0.3(95 \% \mathrm{Cl}-1.2$ to 1.8$)$ & 0.7 & $-0.3(95 \% \mathrm{Cl}-2.1$ to 1.5$)$ & 0.7 \\
\hline ED visits in 2016 (mean \pm SD) & $0.7 \pm 1.0$ & $1.7 \pm 2.7$ & 0.3 & $-1.2(95 \% \mathrm{Cl}-2.1$ to -0.2$)$ & 0.01 & $-1.1(95 \% \mathrm{Cl}-2.0$ to -0.3$)$ & 0.01 \\
\hline
\end{tabular}

${ }^{*} p$ Values for data with normal distributions obtained with Student's $t$-test. $p$ Values for non-normal distributes obtained with Mann-Whitney $U$ test. Transfusion (receiving regular scheduled simple or exchange transfusions). Match 1 propensity matched for age, gender, genotype, hydroxyurea use, and 7 day pain impact score. Match 2 propensity matched for age, gender, genotype, hydroxyurea use, and daily opioids dispensed in OME.

$\mathrm{OME}$, oral morphine equivalents.

Daily cannabis users had fewer annual admissions and annual ER visits when propensity matched with others by variables with effects on pain outcomes

As our study is cross-sectional, baseline characteristics between compared groups may be different and this can create risk of bias. For instance, daily users reported worse pain episodes, so they may be expected to have higher admission or ER visit rates than others, yet they did not show this. However, if people with SCD and severe pain use daily cannabis and achieve pain relief they may have similar admission and ER visit rates to patients with less pain. To attempt to reduce possible confounding bias we used propensity score matching. Propensity matching creates a score based on the variables that may be causing bias, and then compares the treatment group with members of the control group with similar scores. ${ }^{30,31}$ We performed two

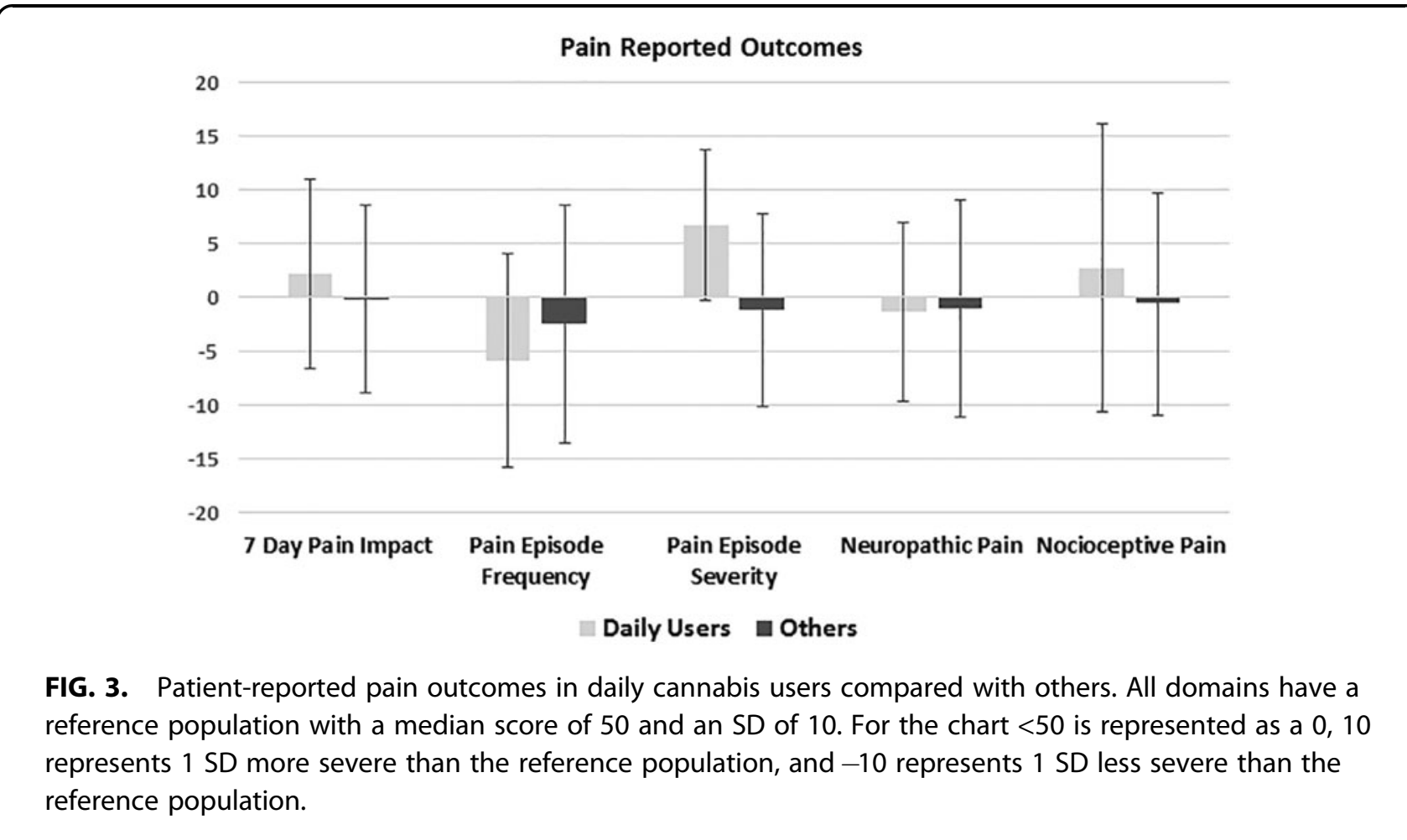




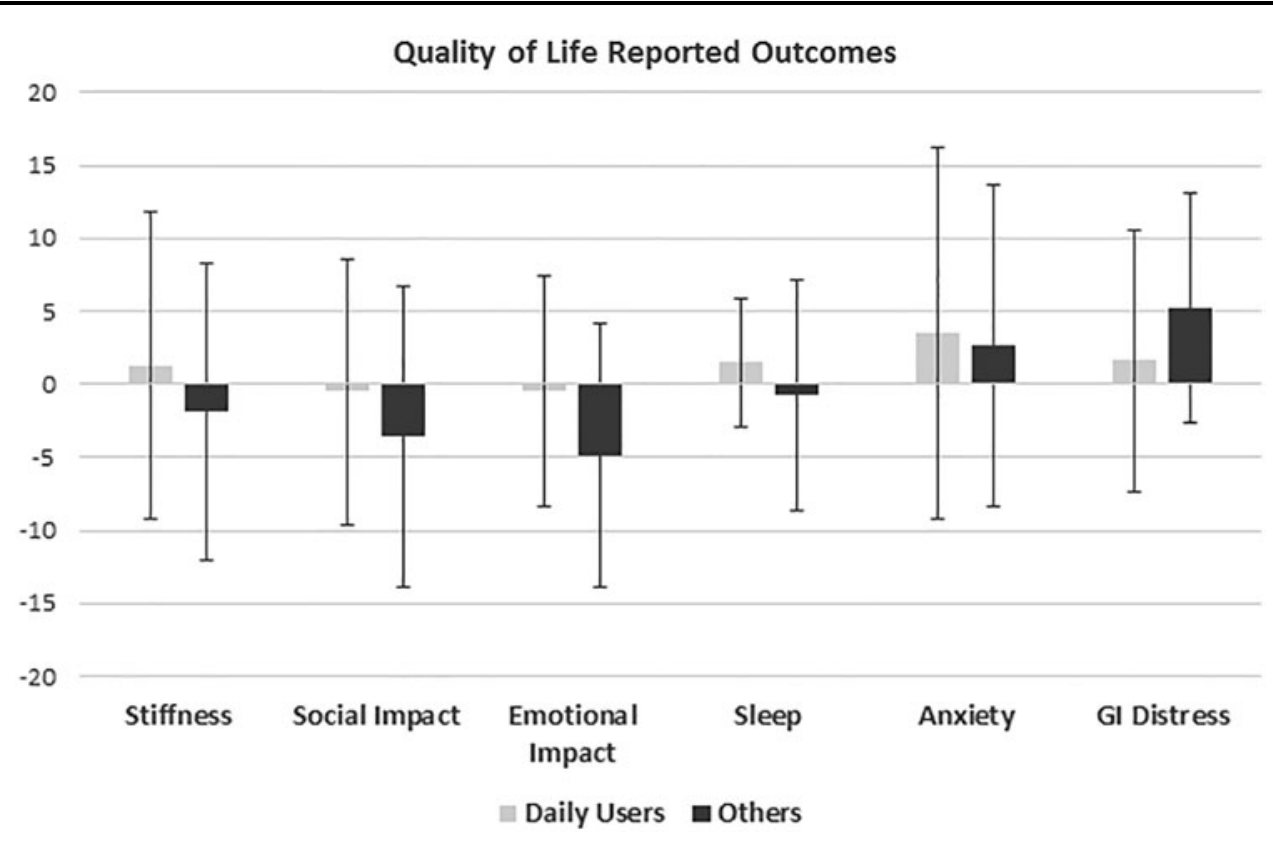

FIG. 4. Patient reported quality-of-life outcomes in daily cannabis users compared with others. All domains have a reference population with a median score of 50 and an SD of 10 . For the chart $<50$ is represented as a 0,10 represents 1 SD more severe than the reference population, and -10 represents 1 SD less severe than the reference population.

matches with variables known to effect pain outcomes. First, we matched for age, gender, SCD genotype, hydroxyurea use, and pain impact score as a measure of daily pain. In this model, compared with others daily users had 1.8 fewer annual admissions $(p=0.01)$ and 1.2 fewer annual ER visits $(p=0.01)$ and showed similar amounts of opioids dispensed and length of stay (Table 1). Second, we matched for age, gender, SCD genotype, hydroxyurea use, and daily opioids dispensed as a measure of daily pain. In this model, compared with others daily users had 2.5 fewer annual admissions $(p=0.02)$ and 1.1 fewer annual ER visits $(p=0.01)$ and again had similar length of stay in days (Table 1 ).

\section{Discussion}

In this descriptive cross-sectional study of patients with SCD, we compared daily cannabis users with others. Daily users reported more severe pain crises yet had lower rates of admission and ER use when compared to others with similar pain and disease severity using propensity matching.

We compared daily cannabis users with all others. Evidence from prior studies examining reasons people use cannabis shows that medical users are more likely to use cannabis daily. ${ }^{29}$ When we examined reported reasons for using cannabis we found no differences between daily user and infrequent users. However, when we examined pain outcomes both infrequent and nonusers of cannabis had scores similar to the mean of the original validation cohorts, and daily cannabis users had more severe scores for pain crisis. We also noted that others had larger standard deviations in admissions (4.1 vs. 1.5$)$ and ER visits (2.7 vs. 1.0$)$ than daily users (Table 1). Thus, the others group may be a heterogenous group made of patients with a mix of severe pain and less severe pain, whereas daily users as a more homogenous group in regard to pain severity. Therefore, we suggest that daily cannabis users are a distinct group from both infrequent and nonusers, and that daily cannabis use is associated with more severe pain crisis.

If the aforementioned is true, then to examine the effects of cannabis on pain it is necessary to compare daily users with a group that has similar pain severity. Although previous cross-sectional studies have not shown that cannabis use was associated with a reduction in health care utilization, this may be due to confounding. If cannabis users have more severe pain 
outcomes (which drive their cannabis use), and if cannabis has an analgesic effect, then the true effect of cannabis use on pain outcomes would be confounded. People with SCD with more severe pain would be expected to have higher rates of hydroxyurea use, narcotic use, and health care utilization, but if cannabis use is analgesic then cannabis users with severe pain may have similar rates of narcotic use and health care utilization to those with less severe pain and thus also have similar rates of hydroxyurea prescriptions. We addressed this possible confounding by adjusting for pain severity using propensity score matching. We matched on variables that would affect both baseline pain severity and pain outcomes, including age, gender, SCD genotype, hydroxyurea use, and either 7-day pain impact or daily opioids dispensed. We chose 7-day pain impact and daily opioids dispensed as these were most representative of average daily pain and we were examining daily cannabis users. In both models daily cannabis use was associated with fewer hospital admissions and fewer ER visits, but no change in daily opioids dispensed. Thus, we posit that cannabis may improve pain outcomes in SCD.

Some studies in other diseases have shown that cannabinoids may be effective in reducing opioid use, but we did not see this in our study. ${ }^{9,10,32}$ Pain in SCD is somewhat unique as it consists both of chronic daily pain and episodes of pain exacerbation in pain crisis. A systematic review of cannabinoids in experimental provoked pain studies published in JAMA Psychiatry suggested that cannabinoids reduce the affective component (pain unpleasantness and impact) of provoked pain. ${ }^{33}$ Anecdotally, patients in our clinic noted that cannabis allowed them to "tolerate pain better." We wonder if we may have seen that daily cannabis users had fewer admissions and ER visits due to the ability of cannabis to increase pain tolerance allowing patients to endure pain crisis at home.

Our study is limited by the cross-sectional design, so we are unable to determine the directionality of the association between exposure and outcome. We used propensity matching to minimize confounding bias; however, this only accounts for known sources of biases, and there may be other determinants of pain in SCD that we failed to adjust for. Daily cannabis users may have reduced health care utilization for reasons not related to pain that we did not address in this study. Our subjects also used varying preparations and amounts of cannabis with varying cannabinoid contents and we were unable to obtain what formulations they used. Illicit cannabis may also be laced with other substances. However, this is a limitation for all noncontrolled cannabis and cannabinoid studies. We are at risk for selection bias. We offered enrollment in our study to a random sample of all patients in our clinic and the majority accepted; however, patients who present more frequently to clinic (likely due to having more severe disease) are more likely to have been offered enrollment. Finally, patients who do not utilize the clinic for care would not have been offered enrollment.

We posit that people with SCD with severe pain are more likely to use daily cannabis due to its analgesic properties. This would explain why daily users reported more severe pain crises yet had fewer admissions and ER visits after propensity matching was performed. A trial of inpatient inhaled cannabis for pain in adults with SCD has been conducted (NCT01771731) and a trial of dronabinol (an oral cannabinoid) for pain in adults with SCD is being conducted (NCT03978156). Further randomized trials and larger prospective trials assessing the impact of cannabinoids on pain in SCD in a clinical setting should be performed.

\section{Acknowledgments}

The authors thank the patients at the Yale New Haven Hospital Adult Sickle Cell Program for their kind participation, Jonathan Spodick and Joanna Cole for their essential help in caring for and enrolling subjects, and the teachers and participants of the 2017/2018 National Clinical Scholars Statistics Course for their input.

\section{Disclosure Statement}

M.D. is employed by Regeneron Pharmaceuticals. This relationship was not related to the development of this article. All other authors report no relevant disclosures/ competing interests.

\section{Funding Information}

This article received funding from the NIH/NHLBI, grant number 5T32HL007974-17.

\section{Supplementary Material}

Supplementary Figure S1

Supplementary Figure S2

\section{References}

1. Brousseau DC, Panepinto JA, Nimmer M, et al. The number of people with sickle-cell disease in the United States: national and state estimates. Am J Hematol. 2010;85:77-78.

2. Brandow AM, Farley RA, Panepinto JA. Neuropathic pain in patients with sickle cell disease. Pediatr Blood Cancer. 2014;61:512-517.

3. Brandow AM, Zappia KJ, Stucky CL. Sickle cell disease: a natural model of acute and chronic pain. Pain. 2017;158(Suppl 1):S79-S84. 
4. Mucke M, Phillips T, Radbruch L, et al. Cannabis-based medicines for chronic neuropathic pain in adults. Cochrane Database Syst Rev. 2018;3: Cd012182.

5. Whiting PF, Wolff RF, Deshpande S, et al. Cannabinoids for medical use: a systematic review and meta-analysis. JAMA. 2015;313:2456-2473.

6. Abrams DI. The therapeutic effects of cannabis and cannabinoids: an update from the National Academies of Sciences, Engineering and Medicine report. Eur J Intern Med. 2018;49:7-11.

7. Bachhuber MA, Saloner B, Cunningham CO, et al. Medical cannabis laws and opioid analgesic overdose mortality in the United States, 1999-2010. JAMA Intern Med. 2014;174:1668-1673.

8. Shi Y. Medical marijuana policies and hospitalizations related to marijuana and opioid pain reliever. Drug Alcohol Depend. 2017;173:144-150.

9. Wen $\mathrm{H}$, Hockenberry JM. Association of medical and adult-use marijuana laws with opioid prescribing for medicaid enrollees. JAMA Intern Med. 2018;178:673-679.

10. Bradford AC, Bradford WD. Medical marijuana laws reduce prescription medication use in medicare part D. Health Aff. 2016:35:1230-1236.

11. National Conference of State Legislatures State Medical Marijuana Laws. 2018. https://www.ncsl.org/research/health/state-medical-marijuanalaws.aspx

12. Howard J, Anie KA, Holdcroft A, et al. Cannabis use in sickle cell disease: a questionnaire study. Br J Haematol. 2005;131:123-128.

13. Knight-Madden J, Lewis N, Hambleton IR. The prevalence of marijuana smoking in young adults with sickle cell disease: a longitudinal study. West Indian Med J. 2006;55:224-227.

14. Ballas SK. The use of cannabis by patients with sickle cell disease increased the frequency of hospitalization due to Vaso-Occlusive Crises. Cannabis Cannabinoid Res. 2017;2:197-201.

15. Roberts JD, Spodick J, Cole J, et al. Marijuana use in adults living with sickle cell disease. Cannabis Cannabinoid Res. 2018;3:162-165.

16. Azofeifa A, Mattson ME, Schauer G, et al. National Estimates of Marijuana Use and Related Indicators-National Survey on Drug Use and Health, United States. MMWR Surveill Summ. 2016;65:1-25. doi: 10.15585/ mmwr.ss6511a1.

17. Ware MA, Doyle CR, Woods $R$, et al. Cannabis use for chronic non-cancer pain: results of a prospective survey. Pain. 2003;102:211-216.

18. Statistics $\mathrm{CfBH}$, Quality. 2015 National Survey on Drug Use and Health: Methodological Summary and Definitions. Substance Abuse and Mental Health Services Administration, Rockville, MD, 2016.

19. Knight-Madden J, Lewis N, Hambleton IR. The prevalence of marijuana smoking in young adults with sickle cell disease: a longitudinal study. West Indian Med J. 2006;55:224-227.

20. Curtis SA, Danda N, Etzion Z, et al. Elevated steady state WBC and platelet counts are associated with frequent emergency room use in adults with sickle cell anemia. PLoS One. 2015;10:e0133116.

21. Askew RL, Cook KF, Keefe FJ, et al. A PROMIS measure of neuropathic pain quality. Value Health. 2016;19:623-630.

22. Keller S, Yang M, Treadwell MJ, et al. Sensitivity of alternative measures of functioning and wellbeing for adults with sickle cell disease: comparison of PROMIS(R) to ASCQ-Me. Health Qual Life Outcomes. 2017;15:117.

23. Keller SD, Yang M, Treadwell MJ, et al. Patient reports of health outcome for adults living with sickle cell disease: development and testing of the ASCQ-Me item banks. Health Qual Life Outcomes. 2014;12:125.
24. Treadwell MJ, Hassell $K$, Levine $R$, et al. Adult sickle cell quality-of-life measurement information system (ASCQ-Me): conceptual model based on review of the literature and formative research. Clin J Pain. 2014;30: 902-914.

25. Pilkonis PA, Choi SW, Reise SP, et al. Item banks for measuring emotional distress from the Patient-Reported Outcomes Measurement Information System (PROMIS(R)): depression, anxiety, and anger. Assessment. 2011; 18:263-283.

26. Nagaraja V, Hays RD, Khanna PP, et al. Construct validity of the PatientReported Outcomes Measurement Information System gastrointestinal symptom scales in systemic sclerosis. Arthritis Care Res. 2014;66:17251730 .

27. Shaiova L. Demystifying opioid conversion calculations, a guide for effective dosing by Mary Lynn McPherson. J Palliat Med. 2011;14:668.

28. Rotermann M, Page MM. Prevalence and correlates of non-medical only compared to self-defined medical and non-medical cannabis use, Canada, 2015. Health Rep. 2018;29:3-13.

29. Hyman SM, Sinha R. Stress-related factors in cannabis use and misuse: implications for prevention and treatment. J Subst Abuse Treat. 2009;36: 400-413.

30. Rosenbaum PR, Rubin DB. The central role of the propensity score in observational studies for causal effects. Biometrika. 1983;70:41-55.

31. Robins JM, Hernan MA, Brumback B. Marginal structural models and causal inference in epidemiology. Epidemiology. 2000;11:550-560.

32. Vyas MB, LeBaron VT, Gilson AM. The use of cannabis in response to the opioid crisis: a review of the literature. Nurs Outlook. 2018;66: 56-65.

33. De Vita MJ, Moskal D, Maisto SA, et al. Association of cannabinoid administration with experimental pain in healthy adults: a systematic review and meta-analysis. JAMA Psychiatry. 2018;75:1118-1127.

Cite this article as: Curtis SA, Brandow AM, DeVeaux M, Zeltermam D, Devine L, Roberts JD (2020) Daily cannabis users with sickle cell disease show fewer admissions than others with similar pain complaints, Cannabis and Cannabinoid Research 5:3, 255-262, DOI: 10.1089/can.2019.0036.

$\quad$ Abbreviations Used
ANOVA $=$ analysis of variance
ASCQ-Me $=$ Adult Sickle Cell Quality of Life Measurement Information
$\quad$ System
$\mathrm{ER}=$ emergency room
$\mathrm{OME}=$ oral morphine equivalents
$\mathrm{PROMIS}=$ Patient-Reported Outcomes Measurement Information
$\quad$ System
$\mathrm{PROS}=$ patient-reported outcomes
$\mathrm{SCD}=$ sickle cell disease
$\mathrm{SD}=$ standard deviation

